\title{
(D) ORIGINAL RESEARCH \\ Stroke vision, aphasia, neglect (VAN) assessment-a novel emergent large vessel occlusion screening tool: pilot study and comparison with current clinical severity indices
}

\author{
Mohamed S Teleb, ${ }^{1}$ Anna Ver Hage, ${ }^{1}$ Jaqueline Carter, ${ }^{1}$ Mahesh V Jayaraman, ${ }^{2}$ \\ Ryan A McTaggart ${ }^{2}$
}

${ }^{1}$ Neurosciences Department, Banner Health, Mesa, Arizona, USA

${ }^{2}$ Department of Diagnostic Imaging Warren Alpert School of Medicine at Brown University, Rhode Island Hospital, Providence, Rhode Island, USA

\section{Correspondence to} Dr M Teleb,

Neurointerventional Surgery, Stroke and Neurocritical Care, Banner Health, 1502 S Dobson Rd, Suite 203, Mesa, AZ 85202, USA; Mohamed. Teleb@bannerhealth.com

Received 23 October 2015 Revised 14 January 2016 Accepted 19 January 2016 Published Online First 18 February 2016

\begin{abstract}
Background Identification of emergent large vessel occlusion (ELVO) stroke has become increasingly important with the recent publications of favorable acute stroke thrombectomy trials. Multiple screening tools exist but the length of the examination and the false positive rate range from good to adequate. A screening tool was designed and tested in the emergency department using nurse responders without a scoring system.
\end{abstract}

Methods The vision, aphasia, and neglect (VAN) screening tool was designed to quickly assess functional neurovascular anatomy. While objective, there is no need to calculate or score with VAN. After training participating nurses to use it, VAN was used as an ELVO screen for all stroke patients on arrival to our emergency room before physician evaluation and CT scan.

Results There were 62 consecutive code stroke activations during the pilot study. $19(31 \%)$ of the patients were VAN positive and 24 (39\%) had a National Institutes of Health Stroke Scale (NIHSS) score of $\geq 6$. All 14 patients with ELVO were either VAN positive or assigned a NIHSS score $\geq 6$. While both clinical severity thresholds had $100 \%$ sensitivity, VAN was more specific ( $90 \%$ vs $74 \%$ for NIHSS $\geq 6$ ). Similarly, while VAN and NIHSS $\geq 6$ had $100 \%$ negative predictive value, VAN had a $74 \%$ positive predictive value while NIHSS $\geq 6$ had only a $58 \%$ positive predictive value.

Conclusions The VAN screening tool accurately identified ELVO patients and outperformed a NIHSS $\geq 6$ severity threshold and may best allow clinical teams to expedite care and mobilize resources for ELVO patients. A larger study to both validate this screening tool and compare with others is warranted.

\section{INTRODUCTION}

Several recent clinical trials ${ }^{1-5}$ have established embolectomy as the standard of care ${ }^{67}$ for patients with severe stroke who present with emergent large vessel occlusion (ELVO). Unfortunately, inefficient prehospital and early hospital care can delay time to embolectomy (groin puncture), the treatment effect for which is time dependent. ${ }^{8}$ Our systems of care need to be designed to match the disease we are treating so that patients both get to the right place the first time and ELVO team activation is early and appropriate.
Effective and efficient prehospital triage of patients with ELVO is now the holy grail of stroke care delivery innovation. Several stroke assessment tools exist, including the 3 item stroke scale (3I-SS), ${ }^{9}$ Cincinnati Prehospital Stroke Severity Scale (CPSSS), ${ }^{10}$ National Institutes of Health Stroke Scale (NIHSS), ${ }^{11}$ face, arm, speech test (FAST),${ }^{12}$ Los Angeles Motor Scale (LAMS) ${ }^{13}$ legs, eyes, gaze, speech (Texas Stroke Intervention Prehospital Stroke Severity Scale) (LEGS), ${ }^{14}$ Melbourne Ambulance Stroke Screen (MASS), ${ }^{15}$ Medic Prehospital Assessment for Code Stroke (Med PACS), ${ }^{15}$ Ontario Prehospital Stroke Screening (OPSS), ${ }^{16}$ Rapid Arterial oCclusion Evaluation Scale (RACE), ${ }^{17}$ and Recognition Of Stroke In the Emergency Room (ROSIER), ${ }^{15}$ some of which (3I-SS, ${ }^{9}$ CPSSS,${ }^{10}$ LAMS,${ }^{13}$ LEGS,${ }^{14}$ RACE,${ }^{17}$ or severe hemiparesis ${ }^{18}$ ) have been used to specifically screen for ELVO but with limitations. For example, the RACE ${ }^{17}$ scoring system is cumbersome for emergency medical technicians and nurses, takes almost as long as the NIHSS, and requires the user to calculate a score. The 3I-SS, ${ }^{9}$ CPSS, ${ }^{10}$ LAMS, ${ }^{13}$ and RACE $^{17}$ scores require the user to remember the test items; the name does not trigger testing or next steps. Using LAMS $^{13}$ or a single deficit screen such as severe hemiparesis ${ }^{18}$ is a more simple approach but has somewhat limited sensitivity and specificity.

Vision, aphasia, and neglect (VAN) is a novel ELVO screening tool we developed to assess functional neurovascular anatomy. It is quick, reproducible, easy to remember and, while objective, requires no score calculations. We tested the ability of VAN to identify ELVO patients presenting to our center and compared it with an NIHSS threshold of $\geq 6$.

\section{METHODS}

\section{Training}

Our emergency room triage nurses, all of whom are NIHSS certified, were trained in how to perform the VAN assessment screen (table 1) during a $2 \mathrm{~h}$ training session. The training session included how to perform VAN, including visual field testing, gaze assessment, aphasia testing, and neglect testing, with simultaneous stimuli. They also were briefly informed of why early stroke thrombectomy is so important for patient outcome
To cite: Teleb MS, Ver

J Neurolntervent Surg

2017:9:122-126. 
Table 1 Vision, aphasia, neglect emergent large vessel occlusion screening tool

\begin{tabular}{ll}
\hline Stroke VAN & \\
\hline How weak is the & $\square$ Mild (minor drift) \\
patient? & $\square$ Moderate (severe drift-touches or nearly touches \\
Raise both arms up & ground) \\
& $\square$ Severe (flaccid or no antigravity) \\
& $\square$ Patient shows no weakness. Patient is VAN negative \\
(exceptions are confused or comatose patients with dizziness, focal findings, or \\
no reason for their altered mental status then basilar artery thrombus must be \\
considered; CTA is warranted) \\
$\begin{array}{ll}\text { Visual disturbance } & \square \text { Field cut (which side) (4 quadrants) } \\
& \square \text { Double vision (ask patient to look to right then left; } \\
& \text { evaluate for uneven eyes) } \\
& \square \text { Blind new onset } \\
& \square \text { None } \\
\text { Aphasia } & \square \text { Expressive (inability to speak or paraphasic errors); do } \\
& \text { not count sluring of words (repeat and name 2 objects) } \\
& \square \text { Receptive (not understanding or following commands) } \\
& \text { (close eyes, make fist) } \\
& \square \text { Mixed } \\
& \square \text { None } \\
& \square \text { Forced gaze or inability to track to one side } \\
& \square \text { Unable to feel both sides at the same time, or unable } \\
& \text { to identify own arm } \\
& \square \text { lgnoring one side } \\
& \square \text { None }\end{array}$ \\
Neglect &
\end{tabular}

Patient must have weakness plus one or all of the $\mathrm{V}, \mathrm{A}$, or $\mathrm{N}$ to be VAN positive. VAN positive patients had $100 \%$ sensitivity, $90 \%$ specificity, positive predictive value $74 \%$, and negative predictive value $100 \%$ for detecting large vessel occlusion. CTA, CT angiography; VAN, vision, aphasia, and neglect.

and emphasized the number needed to treat versus other emergencies in the emergency department, such as ST elevation myocardial infarction (STEMI). Brain and vascular anatomy, as it relates to VAN, was also taught. Functional anatomy of the cortex, motor homunculous, and internal capsule were reviewed. Lacunar syndromes and why they usually do not have cortical symptoms based on anatomy was also emphasized. Lastly, multiple examples of VAN negative and VAN positive patients were presented, including a pure lacunar patient with an NIHSS score of 12 for face, arm, and leg weakness with dysarthria but no aphasia, vision disturbance, or neglect, with follow-up imaging. Other patient examples included sensory neglect, gaze preference, pure expressive and receptive aphasia, and visual field cut.

After the training was completed, the chair of medicine and chief medical officer approved the pilot quality control study due to our prolonged door to puncture times. A formal institutional review board protocol was approved after quality assurance data showed study feasibility and improved process time.

For VAN positive patients, nurses were instructed to both obtain CT angiography (CTA) at the time of the initial noncontrast CT (NCCT) and activate the endovascular stroke team. If CTA confirmed an ELVO, the patient was triaged to the endovascular suite when the team was ready. Intravenous tissue plasminogen activator (tPA) candidacy for all patients was determined by the stroke team either at CT scan or immediately thereafter. Hemorrhages were excluded and treated appropriately after NCCT.

All VAN negative patients also had acute vascular vessel imaging. VAN negative patients received CTA or an MR angiogram of head and neck after administration of tPA. See figure 1 for our stroke triage process before and after the VAN study protocol.

\section{Performing the VAN examination}

The initial and sine qua non of the VAN examination is to conduct a motor weakness assessment (table 1). Patients are asked to raise both arms up and hold them up for $10 \mathrm{~s}$. If the patient has mild drift, severe weakness, or paralysis, the assessment continues. In their absence, the patient is VAN negative and the assessment ends.

If weakness (mild drift, severe weakness, or paralysis) is observed, the VAN assessments are carried out (table 1). If the patient has weakness and any other positive finding among the vision, aphasia, or neglect category, they are considered VAN positive. While forced gaze to one side might be considered a visual finding among users, we included it within the neglect category. Table 2 compares elements of the VAN ELVO screening with others that have been published.

Figure 1 Stroke process before and after the VAN protocol was initiated. CTA, CT angiography; tPa, tissue plasminogen activator; VAN, vision, aphasia, neglect.
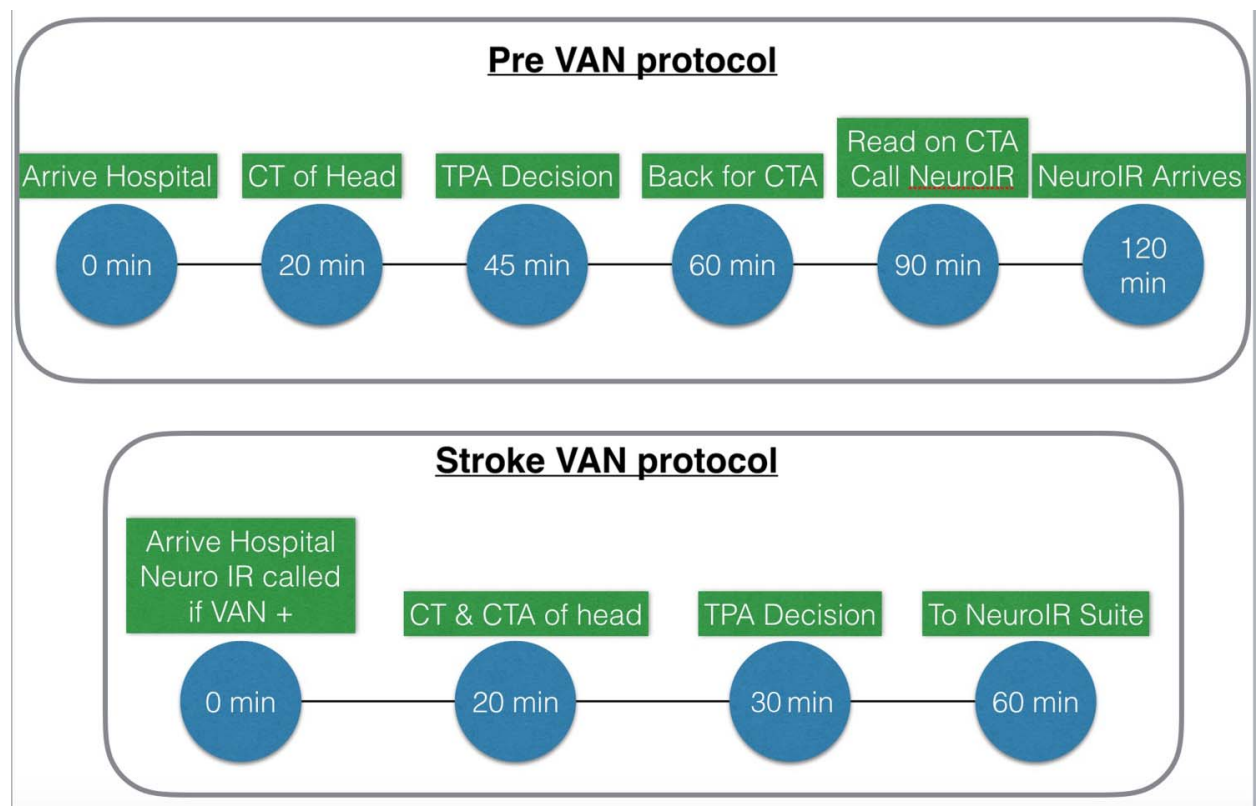


\section{Analysis}

The VAN status of patient was used to compare sensitivity, specificity, positive predictive value, negative predictive value, and accuracy to CTA confirmed ELVO. The same analysis was done using the new guideline recommended (NIHSS of 6). ELVO was defined as thromboembolic occlusion of an M1 segment of the middle cerebral artery (MCA), internal carotid artery, basilar artery, or M2 segment for which embolectomy was considered.

\section{RESULTS}

Sixty-two acute stroke codes were activated during the screening period: 19 were VAN positive and 24 had an NIHSS score of $\geq 6$. The average NIHSS of a VAN positive patient was 18 (range 2-28) and the average for a VAN negative patient was 2 (range $0-7 ; n=9$ had an NIHSS score of $0, n=7$ NIHSS $1, n=8$ NIHSS 2, $\mathrm{n}=1$ NIHSS $3, \mathrm{n}=6$ NIHSS $4, \mathrm{n}=5$ NIHSS $5, \mathrm{n}=6$ NIHSS 6, and $n=1$ NIHSS 7).

Fourteen ELVO patients were considered for embolectomy. All were VAN positive and had a NIHSS score of $\geq 6$. The following embolic occlusions were identified on CTA: 10 M1 MCA (4 left and 6 right), 2 top of basilar into P1 of the posterior cerebral artery (1 right, 1 left), 1 ICA, and 1 M2 MCA occlusion.

Thirty per cent of stroke codes during this period had no weakness, and the VAN screen was completed in $15 \mathrm{~s}$. The average time from symptom onset to stroke code activation and VAN examination was $2 \mathrm{~h}$ during this study period (range $40 \mathrm{~min}$ to $5 \mathrm{~h} 25 \mathrm{~min}$ ).

Table 3 lists the sensitivity, specificity, positive predictive value, negative predictive value, and accuracy of the VAN screening tool. No patient that was VAN negative or determined to have NIHSS $<6$ had an ELVO (both had 100\% negative predictive value). However, VAN had 90\% specificity versus $74 \%$ for NIHSS.

There were five patients who were VAN positive without ELVO (false positive): one patient had multiple distal MCA embolic strokes, another had hypoxia with global weakness and left visual field cut, a third had seizure secondary to an old cortical arteriovenous malformation resection, a fourth had conversion reaction, and one had complex migraine. Unfortunately,

Table 2 Comparison of aspect of the vision, aphasia, neglect emergent large vessel occlusion screening with other screening tools

\begin{tabular}{|c|c|c|c|c|c|c|c|}
\hline Tool & RACE & LEGS & LAMS & Hemiparesis & VAN & 3I-SS & CPSSS \\
\hline \multicolumn{8}{|c|}{ Aspect tested } \\
\hline $\begin{array}{l}\text { Arm } \\
\text { weakness }\end{array}$ & Yes & No & Yes & Yes & Yes & Yes & Yes \\
\hline $\begin{array}{l}\text { Face } \\
\text { weakness }\end{array}$ & Yes & No & Yes & No & No & No & No \\
\hline $\begin{array}{l}\text { Leg } \\
\text { weakness }\end{array}$ & Yes & Yes & No & Yes & No & Yes & No \\
\hline Gaze & Yes & Yes & No & No & Yes & Yes & Yes \\
\hline $\begin{array}{l}\text { Visual field } \\
\text { loss }\end{array}$ & Yes & Yes & No & No & Yes & No & No \\
\hline Neglect & Yes & No & No & No & Yes & No & No \\
\hline $\begin{array}{l}\text { Aphasia/ } \\
\text { speech }\end{array}$ & Yes & Yes & No & No & Yes & No & Yes \\
\hline
\end{tabular}

3I-SS, 3 item stroke scale; CPSSS, Cincinnati Prehospital Stroke Severity Scale; LAMS, Los Angeles Motor Scale; LEGS, legs, eyes, gaze, speech (Texas Stroke Intervention Prehospital Stroke Severity Scale); RACE, Rapid Arterial oCclusion Evaluation Scale; VAN, vision, aphasia, neglect.
Table 3 Sensitivity, specificity, positive predictive value, negative predictive value, and accuracy of vision, aphasia, neglect and National Institutes of Health Stroke Scale for emergent large vessel occlusion

\begin{tabular}{llll}
\hline & Large artery clot & No large artery clot \\
\hline VAN + & 14 & 5 & 19 Total VAN+ \\
VAN- & 0 & 43 & 43 Total VAN- \\
& 14 Large artery clot & 48 No large artery clot & \\
\hline & Large artery clot & No large artery clot \\
NIHSS $\geq 6$ & 14 & 10 & 24 Total \\
NIHSS $<6$ & 0 & 38 & 38 Total \\
& 14 Large artery clot & 48 No large artery clot \\
\hline
\end{tabular}

Positive predictive value of $\mathrm{VAN}=14 / 19=74 \%$; sensitivity $=14 / 14=100 \%$. Positive predictive value of NIHSS $=14 / 24=58 \%$; sensitivity $=14 / 14=100 \%$. Negative predictive value of VAN $=43 / 43=100 \%$; specificity $=43 / 48=90 \%$. Negative predictive value of NIHSS $=38 / 38=100 \%$; specificity $=38 / 48=79 \%$. Accuracy VAN $=57 / 62=92 \%$.

Accuracy NIHSS $=52 / 62=84 \%$.

In addition, the goal of the quality study was to access average door to needle times. Our average time before implementation of VAN was $2 \mathrm{~h} 40 \mathrm{~min}$. The time was reduced to $1 \mathrm{~h} 25$ min when the VAN protocol was employed.

NIHSS, National Institutes of Health Stroke Scale; VAN, vision, aphasia, and neglect.

these were all stroke mimics except for the patient with the multiple distal emboli in the MCA territory.

The interobserver reliability of VAN between the trained nurses and stroke physician was 100\%.

\section{DISCUSSION}

This study demonstrated that VAN is an effective clinical assessment tool by nurses who underwent a $2 \mathrm{~h}$ training session to determine the likelihood of a severe stroke caused by an ELVO. VAN captured all patients that were considered for embolectomy (100\% sensitivity) and only five VAN positive patients were found to not harbor an ELVO (90\% specificity) with $80 \%$ (4/5) being stroke mimics. Furthermore, VAN was easily taught and adopted by nurses in our emergency room and outperformed an NIHSS threshold used by physicians in the emergency room. Because the decay in the treatment effect of embolectomy is profound, earlier treatment is much better, and the ability to use VAN for either pre-hospital or in-hospital triage may be critical to improving ELVO outcome.

While we used VAN as a screening tool in our emergency room we would like to see it used as a prehospital triage tool for ELVO, similar to the way the 3 I-SS, ${ }^{9}$ LAMS,${ }^{13}$ RACE, ${ }^{17}$ LEGS, ${ }^{14}$ and severe hemiparesis ${ }^{18}$ screening tools have been tested and utilized (table 4). Derived from predictive items within the NIHSS, RACE, not surprisingly, had a similar ability to identify an LVO as the NIHSS. ${ }^{17}$ However, in common with the NIHSS, it may be both too cumbersome to administer in the field and have imperfect accuracy. While much easier to employ and also predictive of LVO, LAMS $^{13}$ and 3 I-SS $^{9}$ have been criticized because they may not assess those cortical deficits most predictive of LVO: LAMS ${ }^{13}$ because too few cortical signs are tested and 3I-SS ${ }^{9}$ because it was not derived from the NIHSS ${ }^{15}$. The severe hemiparesis score may have value in the field but this was a small and select cohort of patients that only had this severe hemiparesis so it may not be applicable to all stroke comers. VAN, like 3I-SS, is not completely derived from the NIHSS but does test more cortical functions than both the 3I-SS and LAMS yet retains ease of use. Like LAMS, severe hemiparesis, and 3I-SS, it puts motor deficit as the central feature but only makes you assess cortical 


\section{Ischemic stroke}

Table 4 Emergent large vessel occlusion screening to comparisons

\begin{tabular}{|c|c|c|c|c|c|c|c|}
\hline & RACE & LEGS & LAMS & Hemiparesis & VAN & 3I-SS & CPSSS \\
\hline Need to calculate score & Yes & Yes & Yes & No & No & Yes & Yes \\
\hline No of tests & 6 & 4 & 3 & 1 & $1-4$ & 3 & $3-4$ \\
\hline Length of exam 1-7 (7 is longest) & 7 & 6 & 4 & 1 & 2 & 3 & 5 \\
\hline Positive predictive value (\%) & 42 & 60 & & & 74 & 74 & \\
\hline Sensitivity (\%) & 85 & 69 & 81 & $\begin{array}{l}27-48 \\
\text { multiple etiologies analyzed }\end{array}$ & 100 & 67 & 83 \\
\hline Negative predictive value (\%) & 94 & 86 & & Could not be calculated & 100 & 89 & \\
\hline Specificity (\%) & 68 & 81 & 89 & & 90 & 92 & 40 \\
\hline Type & Prospective & Prospective & Retro & Retro & Prospective & Prospective & Retro \\
\hline Total No of patients analyzed & 357 & 181 & 119 & 45 & 62 & 171 & 303 \\
\hline
\end{tabular}

symptoms if weakness exists, unlike RACE, LEGS, and CPSSS, which force the user to conduct an entire examination every time. In our study, 30\% of stroke codes had no focal motor weakness and the VAN screen took only $15 \mathrm{~s}$. VAN is currently being evaluated by emergency medical services in the field in another small pilot study. Larger numbers as well as interobserver reliability of emergency medical services with nurses and physicians is needed.

In summary, VAN tests multiple cortical divisions of the MCA while always including motor as its central feature. It is always positive when two divisions/deficits are identified with no calculations. There is no need to finish more examinations after two divisions/deficits are identified. VAN uses the best features of previous scales into one tool. Figure 2 shows areas tested for VAN and other LVO screening tools as per area of brain tested. This figure is used in VAN training.

VAN has a number of clinical applications. It can be used in the field by emergency medical services for the pre-notification and activation of code stroke and ELVO teams. Furthermore, it could be used as a screen (by emergency medical services or emergency room triage teams) to determine which patients require CTA at the time of their initial NCCT. Finally, it could

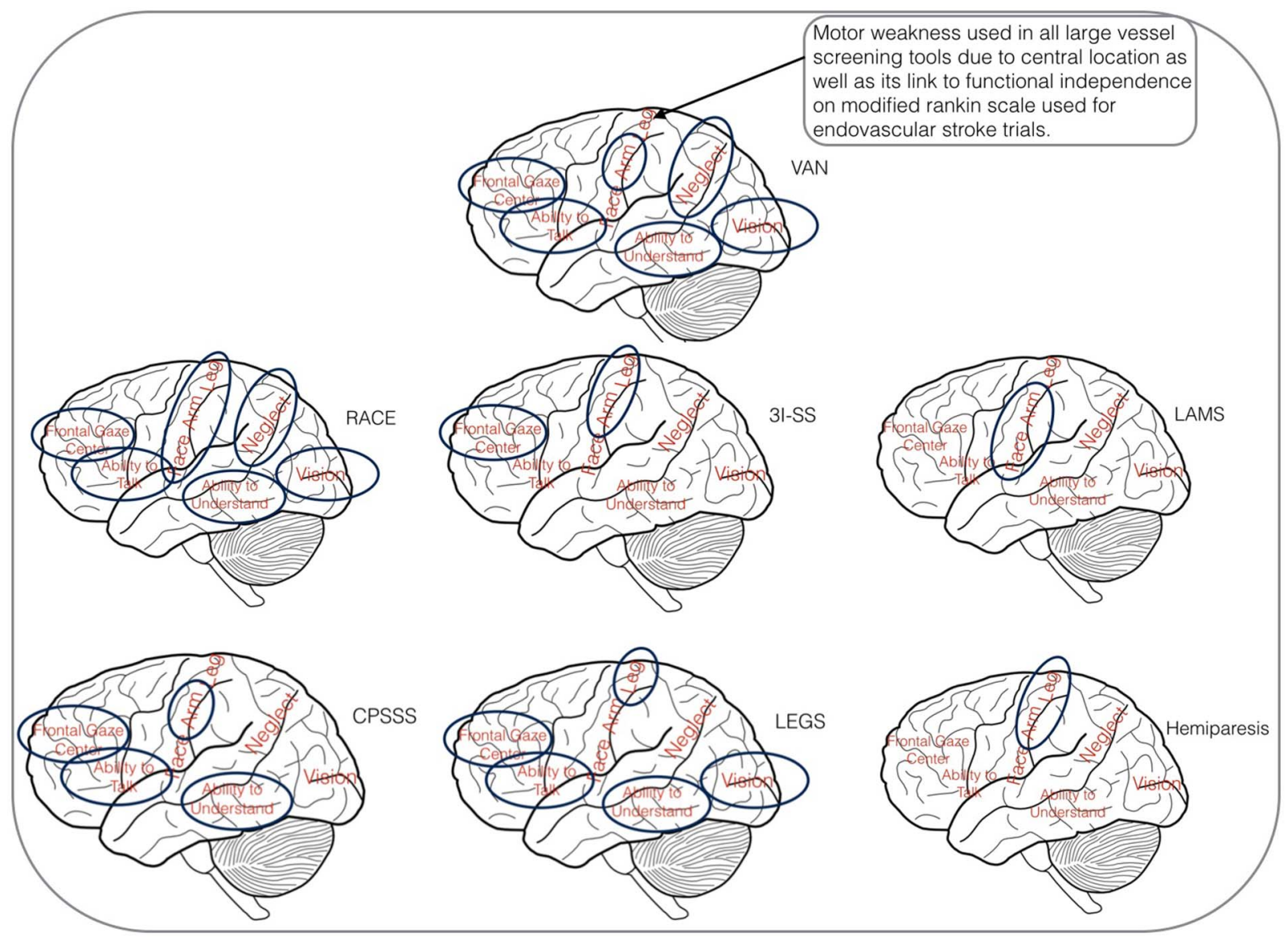

Figure 2 Large vessel occlusion screening tools_-brain view. 3I-SS, 3 item stroke scale; CPSSS, Cincinnati Prehospital Stroke Severity Scale; LAMS, Los Angeles Motor Scale; LEGS, legs, eyes, gaze, speech (Texas Stroke Intervention Prehospital Stroke Severity Scale); RACE, Rapid Arterial oCclusion Evaluation Scale; VAN, vision, aphasia, neglect. 
perhaps be best used as a screening tool with which to determine the appropriate patients to maintain in CT until all treatment decisions are made and initiated. This latter use should improve door to needle times for tPA and door to puncture times for embolectomy, as all downstream stroke processes rely on the results of the initial CT and CTA scans.

The strengths of this study include the fact that the VAN screen was prospectively applied to all stroke codes coming to our emergency room and was compared with a NIHSS threshold of $\geq 6$ which has been suggested as the embolectomy candidacy threshold. ${ }^{7}$ Furthermore, VAN was easily taught and employed. The mnemonic triggers the examination steps and no calculations are required.

A weakness of our study is that it was a single center study of 62 stroke code activations and we may be over-estimating the accuracy of this screening tool. We may have also underestimated the number of clots lysed before vessel imaging for VAN negative patients due to vessel imaging being conducted after administration of tPA. In addition, making a comparison with a similar screening instrument would strengthen our study considerably. However, training the staff to use two scores may confound the ability to assess their utility independently-users may mix the test items. Our study only assessed arm weakness but this is in line with the design of the LAMS and CPSS which were expected to be performed as a seated examination. ${ }^{10} 13$

Our highly trained nurse to stroke physician interobserver reliability of $100 \%$ can be due to the small number of cases. It could also be due to having three levels of freedom with no exact scoring of VAN, allowing a person to miss one item but identify another, and the outcome of VAN positive would not change. In addition to this, the exact degree of weakness was also not scored.

In conclusion, in this prospective small pilot study of code stroke patients, the VAN screening tool was easily taught and adopted, accurately identified patients with ELVO, outperformed a NIHSS threshold, and resulted in early activation of the ELVO team so that door to groin puncture times were improved. Further studies should compare VAN with other prehospital ELVO screens (3P-SS, LAMS, RACE, severe hemiparesis) and quantify the impact on door to needle time and accuracy of ELVO team activation. In addition, larger numbers as well as interobserver reliability of emergency medical services with nurses and physicians is also needed.

Contributors MST conceived the tool and designed the study. AVH and JC helped in data collection. MST and RAM performed the statistical analysis. MST, AVH, JC, MVJ, and RAM reviewed, edited, and approved the paper. MST owns the copyright to VAN.

Competing interests None declared.

Ethics approval The study was approved by the institutional review board.

Provenance and peer review Not commissioned; externally peer reviewed.
Open Access This is an Open Access article distributed in accordance with the Creative Commons Attribution Non Commercial (CC BY-NC 4.0) license, which permits others to distribute, remix, adapt, build upon this work non-commercially, and license their derivative works on different terms, provided the original work is properly cited and the use is non-commercial. See: http://creativecommons.org/ licenses/by-nc/4.0/

\section{REFERENCES}

1 Berkhemer OA, Fransen PSS, Beumer D, et al. A randomized trial of intraarterial treatment for acute ischemic stroke. N Engl J Med 2015;372:11-20.

2 Saver JL, Goyal M, Bonafé A, et al. Stent-retriever thrombectomy after intravenous t-PA vs. t-PA alone in stroke. N Engl J Med 2015;372:2285-95.

3 Goyal M, Demchuk AM, Menon BK, et al. Randomized assessment of rapid endovascular treatment of ischemic stroke. $N$ Engl J Med 2015;372:1019-30.

4 Jovin TG, Chamorro A, Cobo E, et al. Thrombectomy within 8 hours after symptom onset in ischemic stroke. N Engl J Med 2015;372:2296-306.

5 Campbell BCV, Mitchell PJ, Kleinig TJ, et al. Endovascular therapy for ischemic stroke with perfusion-imaging selection. N Engl J Med 2015;372: 1009-18.

6 Jayaraman MV, Hussain MS, Abruzzo T, et al. Embolectomy for stroke with emergent large vessel occlusion (ELVO): report of the Standards and Guidelines Committee of the Society of Neurolnterventional Surgery: table 1. J Neurointerv Surg 2015;7:316-21.

7 Powers WJ, Derdeyn CP, Biller J, et al. 2015 American Heart Association/American Stroke Association Focused Update of the 2013 Guidelines for the Early Management of Patients With Acute Ischemic Stroke Regarding Endovascular Treatment: A Guideline for Healthcare Professionals From the American Heart Association/American Stroke Association. Stroke 2015;46:3020-35.

8 Fransen $\mathrm{P}$, Berkhemer $\mathrm{O}$, Lingsma $\mathrm{H}$. Time to reperfusion and effect of intra-arterial treatment in the MR CLEAN Trial. Int Stroke ... 2015.

9 Singer OC, Dvorak F, Mesnil de Rochemont R, et al. A simple 3-item stroke scale: comparison with the National Institutes of Health Stroke Scale and prediction of middle cerebral artery occlusion. Stroke 2005;36:773-6.

10 Katz BS, McMullan JT, Sucharew H, et al. Design and validation of a prehospital scale to predict stroke severity: Cincinnati Prehospital Stroke Severity Scale. Stroke 2015;46:1508-12.

11 Brott T, Adams HP, Olinger CP, et al. Measurements of acute cerebral infarction: a clinical examination scale. Stroke 1989:20:864-70.

12 Harbison J, Hossain 0 , Jenkinson D, et al. Diagnostic accuracy of stroke referrals from primary care, emergency room physicians, and ambulance staff using the face arm speech test. Stroke 2003;34:71-6

13 Nazliel B, Starkman S, Liebeskind DS, et al. A brief prehospital stroke severity scale identifies ischemic stroke patients harboring persisting large arterial occlusions. Stroke 2008;39:2264-7.

14 Venizelos A, Chen S, Gianatasio R, et al. Abstract T MP59: Texas Stroke Intervention Pre-Hospital Stroke Severity Scale (aka LEGS score): a triaging tool for interventional stroke therapy. Stroke 2014;45:ATMP59-9.

15 Purrucker JC, Hametner C, Engelbrecht A, et al. Comparison of stroke recognition and stroke severity scores for stroke detection in a single cohort. J Neurol Neurosurg Psychiatry 2015;86:1021-8.

16 Brandler ES, Sharma M, Sinert RH, et al. Prehospital stroke scales in urban environments: a systematic review. Neurology 2014;82:2241-9.

17 Pérez de la Ossa N, Carrera D, Gorchs M, et al. Design and validation of a prehospital stroke scale to predict large arterial occlusion: the rapid arterial occlusion evaluation scale. Stroke 2014;45:87-91.

18 Gupta R, Manuel M, Owada K, et al. Severe hemiparesis as a prehospital tool to triage stroke severity: a pilot study to assess diagnostic accuracy and treatment times. J Neurointerv Surg 2016;8:775-7. 